\title{
SIMILARITY SOLUTIONS FOR CONVECTION OF GROUNDWATER ADJACENT TO HORIZONTAL IMPERMEABLE SURFACES WITH AXISYMMETRIC TEMPERATURE DISTRIBUTION
}

\author{
TECHNICAL REPORT NO, 14
}

April 30, 1976

Prepared Under

NATIONAL SCIENCE FOUNDATION

Research Grant No. GI-38319

and

ENERGY RESEARCH AND DEVELOPMENT ADMINISTRATION

Research Grant No. E(04-3)-1093

\section{By}

\section{Ping Cheng}

and

W. C. Chau

Department of Mechanical Engineering

University of Hawai $i$

Honolulu, Hawaii

DISCLAIMER

The This book was pref St ses Government nor any seency thereot, nor sny of their employees, makes any Neither the Unled States Golied, or assumes any legal liability of responsibitity tor the accuracy, warranty, ex, usefulness of any information, apparatus, produc, or procein 10 ary specific represents that its use mould not infringe privately owned commercial product, process, or senvice by trode nam, inconmentation, or favoring by the United not necesserily sonstitute or imply its endorsons. States Government or any agency thereof. The view Government or any agency thereot. 


\section{DISCLAIMER}

This report was prepared as an account of work sponsored by an agency of the United States Government. Neither the United States Government nor any agency Thereof, nor any of their employees, makes any warranty, express or implied, or assumes any legal liability or responsibility for the accuracy, completeness, or usefulness of any information, apparatus, product, or process disclosed, or represents that its use would not infringe privately owned rights. Reference herein to any specific commercial product, process, or service by trade name, trademark, manufacturer, or otherwise does not necessarily constitute or imply its endorsement, recommendation, or favoring by the United States Government or any agency thereof. The views and opinions of authors expressed herein do not necessarily state or reflect those of the United States Government or any agency thereof. 


\section{DISCLAIMER}

Portions of this document may be illegible in electronic image products. Images are produced from the best available original document. 


\section{NOMENCLATURE}

constant defined by Eq. (6a)

specific heat of the convective fluid

dimensionless stream function defined by Eq. (16)

acceleration due to gravity

local heat transfer coefficient

average heat transfer coefficient defined

permeability of the porous medium

thermal conductivity of the porous medium

local Nusselt number, $\mathrm{Nu} r=\mathrm{hr} / \mathrm{k}$

average Nusselt number, $\overline{\mathrm{Nu}}=\overline{\mathrm{h} R} / \mathrm{k}$

pressure

over-all heat transfer rate

local heat transfer rate

$r$

coordinate in the radial direction

R

radius of the impermeable surface

$\mathrm{Ra}$

$$
\text { modified Rayleigh number, } R a=\rho_{\infty} g K \beta\left|T_{W}-T_{\infty}\right| R / \mu \alpha
$$

modified local Rayleigh number, $R_{r}=\rho_{\infty} g K B\left|T_{w}-T_{\infty}\right| r / \mu \alpha$ temperature

velocity component in r-direction

velocity component in z-direction

coordinate in the vertical direction

$\alpha$

equivalent thermal diffusivity

B

coefficient of thermal expansion

$\delta_{m}$ momentum boundary layer thickness 


$\begin{array}{ll}n & \text { dimensionless similarity variable defined by Eq. } \\ \eta_{m} & \text { value of } n \text { at the edge of momentum boundary layer } \\ n_{T} & \text { value of } n \text { at the edge of thermal boundary layer } \\ \theta & \text { dimensionless temperature defined by Eq. (17) } \\ \lambda & \text { constant defined by Eq. (6a) } \\ \mu & \text { viscosity of convective fluid } \\ \rho & \text { density of convective fluid } \\ \psi & \text { stream function } \\ \Psi & \text { dimensionless stream function, } \Psi \equiv \psi / R\left(R a_{r}\right)^{1 / 3}\end{array}$

\section{Subscript}

$\infty$

$w$ condition at infinity

condition at the wall 


\section{ABSTRACT}

The axisymmetric buoyancy-induced groundwater flow adjacent to horizontal impermeable surfaces with wall temperature being a power function of radius is discussed in this paper. With the boundary layer simplifications, the governing non-linear partial differential equations can be transformed into a coupled pair of non-linear ordinary differential equations with two-point boundary conditions that can be integrated numerically by established techniques. Simple algebraic expressions for boundary layer thickness and heat transfer rate are obtained. Applications to free convective flow in a liquid-dominated geothermal system at high Rayleigh number are discussed. 


\section{Introduction}

The prediction of heat transfer rate from a heated or cooled horizontal surface to surrounding groundwater in a subsurface environment has import applications to the assessment of geothermal resources and the design of a geothermal power plant. The problem can be idealized as a horizontal flate plate embedded in a saturated porous medium of infinite extent. It has been recognized for some time that convection in a saturated porous medium and in an incompressible fluid have much in common. Thus, in analogous to the classical free convection problems at high Grashof numbers treated by Stewartson [1] and Gill [2], it can be conjectured that convective heat transfer in a porous medium at high Rayleigh numbers takes place in a thin layer adjacent to the heated and cooled surfaces. Indeed, recent finite difference solutions by Cheng, Yueng, and Lau [3] for free convection in a liquid-dominated goethermal reservoir shows that boundary layer behavior becomes increasingly pronounced in the flow field near the heated or cooled surfaces as the Rayleigh number of the reservoir is increased. The boundary layer approximations have been employed earlier by Wooding [4], McNabb [5], Yih [6] and recently by Cheng and Minkowycz [7] to obtain analytical solutions to a number of free convection problems in a saturated porous medium at high Rayleigh numbers.

In this paper the axisymmetric buoyancy induced flow in a saturated porous medium above a heated or below a cooled horizontal impermeable surface with prescribed wall temperature being a power function of radius is studied. Boundary layer approximations similar to the previous work [4-7] are invoked and similarity solutions are obtained. Numerical integration of the resulting two-point boundary value problem is carried out, and expressions for heat transfer rate and boundary layer thickness are derived. Computations for heat transfer rate and the size of the hot water zone above a heated bedrock with 
$1 \mathrm{~km}$ in radius maintained at different wall temperature distributions are, carried out.

\section{Analysis}

Consider the asymmetric buoyancy flow in a saturated porous medium above a heated horizontal impermeable surface or below a cooled surface with wall temperature being a function of radius. The coordinate system is shown in Fig. 1 where $r$ and $z$ are cylindrical coordinates in horizontal and vertical directions with positive $z$ axis pointing toward the porous medium. If we assume that (i) the convective fluid and the porous medium are everywhere in local thermodynamic equilibrium, (ii) the temperature of the fluid is everywhere below boiling point, (iii) properties of the fluid and the porous medium are constant, and (iv) the Boussinesq approximation is employed, the governing equations are given by

$$
\begin{aligned}
& \frac{\partial}{\partial r}(r u)+\frac{\partial}{\partial z}(r w)=0, \\
& u=-\frac{K}{\mu} \frac{\partial p}{\partial r}, \\
& w=-\frac{K}{\mu}\left(\frac{\partial p}{\partial z} \pm \rho g\right), \\
& \rho=\rho_{\infty}\left[1-\beta\left(T-T_{\infty}\right)\right], \\
& u \frac{\partial T}{\partial r}+w \frac{\partial T}{\partial z}=\alpha\left(\frac{1}{r} \frac{\partial}{\partial r}\left(r \frac{\partial T}{\partial r}\right)+\frac{\partial^{2} T}{\partial z^{2}}\right),
\end{aligned}
$$

where the "+" sign in Eq. (3) refers to the case of a heated impermeable surface facing upward (Fig. 1b) while the "-" sign refers to the case of a 
cooled impermeable surface facing downward (Fig. 1a). In Eqs. (1) to (5), $u$ and $w$ are the velocity components in the horizontal and vertical directions respectively; $\rho, \mu$, and $\beta$ are the density, viscosity and the thermal expansion coefficient of the convecting fluid respectively; $K$ is the permeability of the porous medium; $\alpha=k /\left(\rho_{\infty} C\right)_{f}$ is the equivalent thermal diffusivity with $k$ denoting the thermal conductivity of the saturated porous medium and $\left(\rho_{\infty} C\right)_{f}$ the product of density and specific heat of the convecting fluid; T, $p$ and g are respectively the temperature, pressure and the gravitational acceleration. The subscript "oo" refers to the condition at infinity.

The boundary conditions for the problem are

$$
\begin{array}{lll}
z=0, & T_{w}=T_{\infty} \pm A r^{\lambda}, & w=0, \\
z+\infty, & T=T_{\infty}, & u=0,
\end{array}
$$

where $A>0$ and the " + " and "-" signs in Eq. (6a) are for a heated impermeable surface facing upward and for a cooled impermeable surface facing downward respectively. Eq. (6a) shows that the prescribed wall temperature is a power function of radius from the origin.

The continuity equation is automatically satisfied by introducing the stream function $\psi$ as

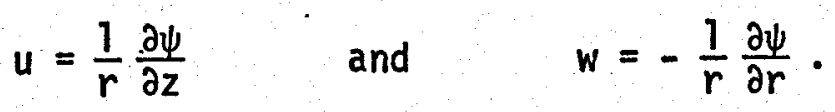

Eliminating $\mathrm{p}$ from Eqs. (2) and (3) by cross differentiation, the resulting equation in terms $\psi$ is

$$
\frac{\partial}{\partial r}\left(\frac{1}{r} \frac{\partial \psi}{\partial r}\right)+\frac{1}{r} \frac{\partial^{2} \psi}{\partial z^{2}}= \pm \frac{K \rho_{\infty} g \beta}{\mu} \frac{\partial T}{\partial r}
$$


Equation (4), in terms of $\psi$, can be rewritten as

$$
\frac{\partial}{\partial r}\left(r \frac{\partial T}{\partial r}\right)+r \frac{\partial^{2} T}{\partial z^{2}}=\frac{1}{\alpha}\left[-\frac{\partial \psi}{\partial z} \frac{\partial T}{\partial r}+\frac{\partial \psi}{\partial r} \frac{\partial T}{\partial z}\right]
$$

The appropriate boundary conditions for Eqs. (9) and (10) are

$$
\begin{array}{ll}
z=0, \quad T_{W}=T_{\infty}+A r^{\lambda}, & \frac{\partial \psi}{\partial r}=0, \\
z+\infty, \quad T=T_{\infty}, & \frac{\partial \psi}{\partial z}=0 .
\end{array}
$$

III. Similarity Solution

If we assume that the boundary layer behavior exists, Eqs. (9) and (10) can be approximated by

$$
\begin{aligned}
& \frac{1}{r} \frac{\partial^{2} \psi}{\partial z^{2}}= \pm \frac{K p_{\infty} g \beta}{\mu} \frac{\partial T}{\partial r} \\
& \frac{\partial^{2} T}{\partial z^{2}}=\frac{1}{\alpha}\left(\frac{1}{r} \frac{\partial \psi}{\partial r} \frac{\partial T}{\partial z}-\frac{1}{r} \frac{\partial \psi}{\partial z} \frac{\partial T}{\partial r}\right)
\end{aligned}
$$

To seek similarity solutions to Eqs. (13) and (14) with boundary conditions (11) and (12), we now introduce the following dimensionless variables

$$
\begin{aligned}
& \eta=\left[\frac{K \rho_{\infty} g \beta A}{\mu \alpha}\right]^{1 / 3} z r(\lambda-2) / 3=\left(R a_{r}\right)^{1 / 3} \frac{z}{r}, \\
& \psi=\alpha\left[\frac{K \rho_{\infty} g A \beta}{\mu \alpha}\right]^{1 / 3} r^{(4+\lambda) / 3} f(n)=\alpha\left(R a_{r}\right)^{1 / 3} r f(n), \\
& \theta(n)=\left(T-T_{\infty}\right) /\left(T_{W}-T_{\infty}\right),
\end{aligned}
$$

where $R_{r}=\rho_{\infty} K g \beta\left|T_{W}-T_{\infty}\right| r / \mu \alpha$ is the modified local Rayleigh number. 
In terms of new variables, it can be shown that the velocity components are given by

$$
\begin{aligned}
& u=\alpha\left[\frac{K \rho_{\infty} g A B}{\mu \alpha}\right]^{2 / 3} r^{(2 \lambda-1) / 3} f^{\prime}(\eta), \\
& w=-\alpha\left[\frac{K \rho_{\infty} g A B}{\mu \alpha}\right]^{1 / 3} \frac{r}{3}(\lambda-2) / 3\left[(\lambda-2) \eta f^{\prime}+(4+\lambda) f\right] .
\end{aligned}
$$

and the governing equations (13) and (14) and their appropriate boundary conditions are

$$
\begin{aligned}
& f^{\prime \prime}+\lambda \theta+\frac{(\lambda-2)}{3} n \theta^{\prime}=0, \\
& \theta^{\prime \prime}-\lambda \theta f^{\prime}+\frac{(4+\lambda)_{f \theta^{\prime}}=0,}{3}
\end{aligned}
$$

and

$$
\begin{array}{ll}
\theta(0)=1, & f(0)=0, \\
\theta(\infty)=0, & f^{\prime}(\infty)=0 .
\end{array}
$$

IV. Results and Discussion

The boundary layer approximations used in obtaining Eqs. (20) and (21) are valid if (i) $\frac{\partial}{\partial z} \gg \frac{\partial}{\partial r}$ and (ii) $w \ll u$. From Eq. (15), it follows that $z / r=$ $0\left(\mathrm{Ra}_{r}^{-1 / 3}\right)$. Furthermore, the ratio of Eqs. (19) and Eq. (18) gives $w / u=$ $O\left(\operatorname{Ra}_{r}^{-1 / 3}\right)$. Thus, the two conditions are satisfied if $\mathrm{Ra}_{r}$ is large. Consequently, the boundary layer approximations are not expected to be valid near $r=0$. To find the range of $\lambda$ for which the problem is physically realistic, we follow the approach discussed by Gebhart [8] by examining Eq. (18) and the expressions for the boundary layer thickness which can be obtained from Eq. (15) to give

$$
\frac{\delta_{m}}{r}=\frac{n_{m}}{\left(R a_{r}\right)^{1 / 3}} \text { and } \quad \frac{\delta_{T}}{r}=\frac{n_{T}}{\left(R_{r}\right)^{1 / 3}} \text {, }
$$


where $n_{m}$ and $n_{T}$ denote values of $n$ where $u / u_{r}$ or $\theta$ has a value of 0.01 . Since the wall temperature differs from that of the surrounding fluid at $r=0$, both $u$ and $\delta$ the boundary layer thickness must be increasing or at least constant with respect to $r$ [8]. It follows from Eqs. (18) and (24) that these conditions are satisfied if $1 / 2 \leq \lambda \leq 2$.

Numerical integration for the two-point boundary value problem, Eqs. (20)(23), can be obtained using the Runge-Kutta method by first converting to an initial value problem with a systematic guessing of the values of $f^{\prime}(0)$ and $\theta^{\prime}(0)$ by the shooting technique. Numerical results for selected $\lambda$ in the range of $1 / 2 \leq \lambda \leq 2$ are presented in Figs. 2 through 7 . The values of $\eta_{m}$ and $\eta_{T}$ in Eqs. (24) can be obtained from Figs. 2 and 3. These values are tabulated in Table 1, which shows that the momentum and thermal boundary layer thickness are of the same order of magnitude.

With the aid of Eqs. (15) and (17), the local surface heat flux is given by

$$
q=-k\left(\frac{\partial T}{\partial y}\right)=k A^{4 / 3}\left[\frac{\rho_{\infty} K g \beta}{\mu \alpha}\right]^{1 / 3} r^{2(2 \lambda-1) / 3}\left[-\theta^{\prime}(n)\right],
$$

where the values of $\left[-\theta^{\prime}(n)\right]$ is plotted in Fig. 4. The local surface heat flux is given by Eq. (25) with $\eta=0$ which shows that the local surface heat flux is constant for $\lambda=1 / 2$ and increases with respect to $r$ for other values of $\lambda$. From the definition of the local Nusselt number $N u_{r}=\frac{h r}{k}=\frac{g r}{k\left(T_{w}-T_{\infty}\right)}$ with $h$ denoting the local heat transfer coefficient, Eq. (25) can be rewritten as

$$
\frac{N u_{r}}{\left(R a_{r}\right)^{1 / 3}}=\left[-\theta^{\prime}(0)\right]
$$

where the value of $\left[-\theta^{\prime}(0)\right]$ for selected $\lambda$ is tabulated in Table 1.

The overall surface heat transfer rate for a circular surface with a radius $R$ can be computed from 


$$
Q=\int_{0}^{R} q(r) 2 \pi r d r
$$

which can be integrated after the substitution of Eq. (25) to give

$$
Q=\frac{3 \pi}{2(\lambda+1)^{k}} A^{4 / 3} \cdot\left[\frac{\rho_{\infty} K g \beta}{\mu \alpha}\right]^{1 / 3} R^{4(\lambda+1) / 3}\left[-\theta^{\prime}(0)\right] .
$$

The average Nusselt number is defined by $\overline{\mathrm{Nu}}=\overline{\mathrm{h}} R / \mathrm{k}$ where the average heat transfer coefficient $\bar{h}$ depends on the choice of the temperature difference between the wall and the temperature of the fluid away from the wall. Consider the temperature difference based on the mean temperature difference defined by

$$
\left(\overline{T_{W}-T_{\infty}}\right)=\frac{1}{\pi R^{2}} \int_{0}^{R}\left(T_{W}-T_{\infty}\right) 2 \pi R d r=\frac{2}{(\lambda+2)} A R^{\lambda}=\frac{2}{(\lambda+2)}\left(T_{W}-T_{\infty}\right) .
$$

Also from the definition of the average heat transfer coefficient, we have

$$
Q=\bar{h}\left(\overline{T_{W}-T_{\infty}}\right) \pi R^{2}
$$

Equating Eq. (28) with Eq. (30) and from the definition of the average Nusselt number defined earlier, we have

$$
\frac{\overline{N u}}{\mathrm{Ra}^{1 / 3}}=\frac{3(\lambda+2)^{4 / 3}}{2^{7 / 3}(\lambda+1)}\left[-\theta^{\prime}(0)\right],
$$

where $\mathrm{Ra} \equiv\left|\overline{\mathrm{T}_{W}-\mathrm{T}_{\infty}}\right| \rho_{\infty} g B K R / \mu \alpha$.

To obtain the induced pressure along the wall, we equate Eqs. (2) and (18) and integrate along the line $z=0$ to get

$$
p(r, 0)=-\frac{3 \alpha \mu}{2(\lambda+1)}\left[\frac{K \rho_{\infty} g B A}{\mu \alpha}\right]^{2 / 3} f^{\prime}(0) r(2 \lambda+1) / 3,
$$


which shows that the induced pressure is increasingly more negative with ' $r$.

It is noted that although $u \rightarrow 0$ outside the momentum boundary layer, the vertical velocity component outside the boundary layer, in general, has a nonzero value given by

$$
w_{\infty}=-\alpha\left[\frac{K \rho_{\infty} g A B}{\mu \alpha}\right]^{1 / 3} r^{(\lambda-2) / 3}\left(\frac{4+\lambda}{3}\right) f(\infty),
$$

which is obtained from Eq. (19) with the aid of Eq. (23b). Equation (33) shows that $w_{\infty}$ is negative for the range of $\lambda$ considered since $f(\infty)$ is a non-zero negative value as shown in Fig. 5 . The variation of the dimensionless vertical velocity is plotted in Fig. 6 , where it is shown that its magnitude increases from zero to a finite value as $\eta$ is increased. To plot the convective pattern of groundwater, we note from Eqs. (15) and (16) that $\Psi=\Psi\left[\frac{r}{R}, \frac{Z}{R}\left(R a_{r}\right)^{1 / 3} ; \lambda\right]$ where $\Psi \equiv \psi / R\left(R_{r}\right)^{1 / 3}$ is the dimensionless stream function. A representative plot of streamlines is presented in Fig. 7, where the dimensionless coordinates are $\frac{r}{R}$ and $\frac{z}{R}\left(R a_{r}\right)^{1 / 3}$.

To gain some insights of the magnitudes of various physical quantities in a geothermal application, consider an upward facing heated impermeable surface of radius $1 \mathrm{~km}$ with wall temperature increasing from $288 \mathrm{~K}$ at $r=0$ to $573 \mathrm{~K}$ at $r=1 \mathrm{~km}$. The wall temperature distribution for different values of $\lambda \cdot$ is sketched in Fig. 8. For numerical computations the following physical properties are used: $\beta=2.8 \times 10^{-4} / \mathrm{K}, \rho_{\infty}=0.92 \times 10^{6} \mathrm{~g} / \mathrm{m}^{3}, C=4.2 \times 10^{3}$ Joule $/ \mathrm{Kg}-\mathrm{K}$, and $k=2.4$ Watt/m-K. The value of permeability reported in the literature differs greatly, ranging from $10^{-14} \mathrm{~m}^{2}$ given by McNabb [5] for the rock formation at Wairakei, New Zealand, to about $10^{-10} \mathrm{~m}^{2}$ given by Soroos [9] for the island of Hawaii. The value of $\mu$ is a strong function of temperature varying from 
$0.54 \times 10^{-3}$ Newton-sec $/ \mathrm{m}^{2}$ at $288^{\circ} \mathrm{K}$ to $0.042 \times 10^{-3}$ Newton-sec $/ \mathrm{m}^{2}$ at $573^{\circ} \mathrm{K}$. If the values of $k=10^{-12} \mathrm{~m}^{2}$ and $\mu=0.54 \times 10^{-3}$ Newton-sec $/ \mathrm{m}^{2}$ are used, the boundary layer thickness at $r=1 \mathrm{~km}$ and the total heat transfer rate for selected $\lambda$ are tabulated in Table 2. Since at a fixed location, the prescribed wall temperature decreases as $\lambda$ is increased (Fig. 8), consequent7y, both the boundary layer thickness and the total heat transfer rate decrease as $\lambda$ is increased (Table 2). If the values of $\mu=0.042 \times 10^{-3}$ Newton-sec $/ \mathrm{m}^{2}$ and $\mathrm{K}=10^{-10} \mathrm{~m}^{2}$ are used, the boundary layer thickness would be considerably thinner with an associated increase in heat transfer rate.

\section{Concluding Remarks:}

Although the foregoing analysis is discussed in terms of the convection of groundwater adjacent to horizontal impermeable surfaces, the results are applicable to a wide range of geophysical and engineering problems whenever they can be idealized as a horizontal flat plate embedded in a saturated porous medium. The analysis is based on the boundary layer approximations which are valid for large Rayleigh numbers. The simple algebraic expressions for total heat transfer rate and boundary layer thickness (or the hot water zone) are useful for a quick estimate of geothermal resources.

\section{Acknowledgment}

This study is part of the Hawaii Geothermal Project funded in part by the RANN program of the National Science Foundation of the United States (Grant No. GI-38319), the Energy Research and Development Administration of the United States (Grant No. E(04-3)-1093), and by the State and County of Hawaii. 


\section{REFERENCES}

1. Stewartson, K., "On the Free Convection from a Horizontal Plate," ZAMP, v. 9, 276-281 (1958).

2. Gill, W.N., Zeh, D.W., and Casal, E.D., "Free Convection on a Horizontal Plate," ZAMP, v. 16, 539-541 (1965).

3. Cheng, P., Yueng, K.C., and Lau, K.H., "Numerical Solutions for Steady Free Convection in Island Geothermal Reservoirs." To appear in Proceedings of 1975 International Seminar, on Future Energy Production -- Heat and Mass Transfer Problems.

4. Wooding, R.A., ."Convection in a Saturated Porous Medium at Large Rayleigh Number or Peclet Number," J. Fluid Mechanics, v. 15, 527-544 (1963).

5. McNabb, A., "On Convection in a Saturated Porous Medium, " Proceedings of the 2nd Australian Conference on Hydraulics and Fluid Mechanics, C161-C171 (1965).

6. Yih, C.S., Dynamics of Nonhomogeneous Fluids, Macmillan Publishing Co., Inc., New York (1965).

7. Cheng, P. and Minkowycz, W.J., "Similarity Solutions for Free Convection About a Dike," Hawaii Geothermal Project, Technical Report No. 10, October 1975.

8. Gebhart, B., Heat Transfer, McGraw-Hi11 Book Company (1971).

9. Soroos, R.L., "Determination of Hydraulic Conductivity of Some Oahu Aquifers with Step-Draw Down Test Data," M.S. Thesis, Department of Geology and Geophysics, University of Hawaii, May 1973. 
TABLE 1. Values of $-\theta^{\prime}(0), \eta_{T}$ and $\eta_{m}$ for selected values of $\lambda$

\begin{tabular}{|l|l|l|l|}
\hline$\lambda$ & $-\theta^{\prime}(0)$ & $n_{\mathrm{T}}$ & $\ldots n_{\mathrm{m}}$ \\
\hline 0.5 & 0.9305 & 3.469 & 4.267 \\
1.0 & 1.162 & 3.255 & 3.748 \\
1.5 & 1.379 & 3.069 & 3.299 \\
2.0 & 1.587 & 2.909 & 2.909 \\
\hline
\end{tabular}

TABLE 2. Values of $\delta_{T}, \delta_{m}$ at $r=1 \mathrm{~km}$ and $Q$ for selected values of $\lambda$

\begin{tabular}{|c|c|c|c|}
\hline$\lambda$ & $\left(\delta_{T}\right)_{r=1 \mathrm{~km}}$ & $\left(\delta_{\mathrm{m}}\right)_{\mathrm{r}=1 \mathrm{~km}}$ & $\mathrm{Q}$ \\
\hline 0.5 & $268.9 \mathrm{~m}$ & $330.7 \mathrm{~m}$ & $25.8 \mathrm{MW}$ \\
1.0 & $252.3 \mathrm{~m}$ & $290.5 \mathrm{~m}$ & $24.2 \mathrm{MW}$ \\
1.5 & $237.9 \mathrm{~m}$ & $255.7 \mathrm{~m}$ & $22.9 \mathrm{MW}$ \\
2.0 & $225.5 \mathrm{~m}$ & $225.5 \mathrm{~m}$ & $21.9 \mathrm{MW}$ \\
\hline
\end{tabular}




\section{LIST OF FIGURES}

1. Coordinate System

2. Values of $\theta$ Versus $n$ for Selected Values of $\lambda$

3. Dimensionless Velocity Distribution Versus $n$ for Selected Values of $\lambda$

4. Values of $-\theta^{\prime}$ Versus $n$ for Selected Values of $\lambda$

5. $f$ Versus $n$ for. Selected Values of $\lambda$

6. Dimensionless Vertical Velocity Versus $n$ for Selected Values of $\lambda$

7. Streamlines Distribution for a Heated Impermeable Surface Facing Upward

8. Sketch of the Wall Temperature Distribution for Selected Values of $\lambda$ 


$$
T_{w}=T_{\infty}-A r^{\lambda}
$$
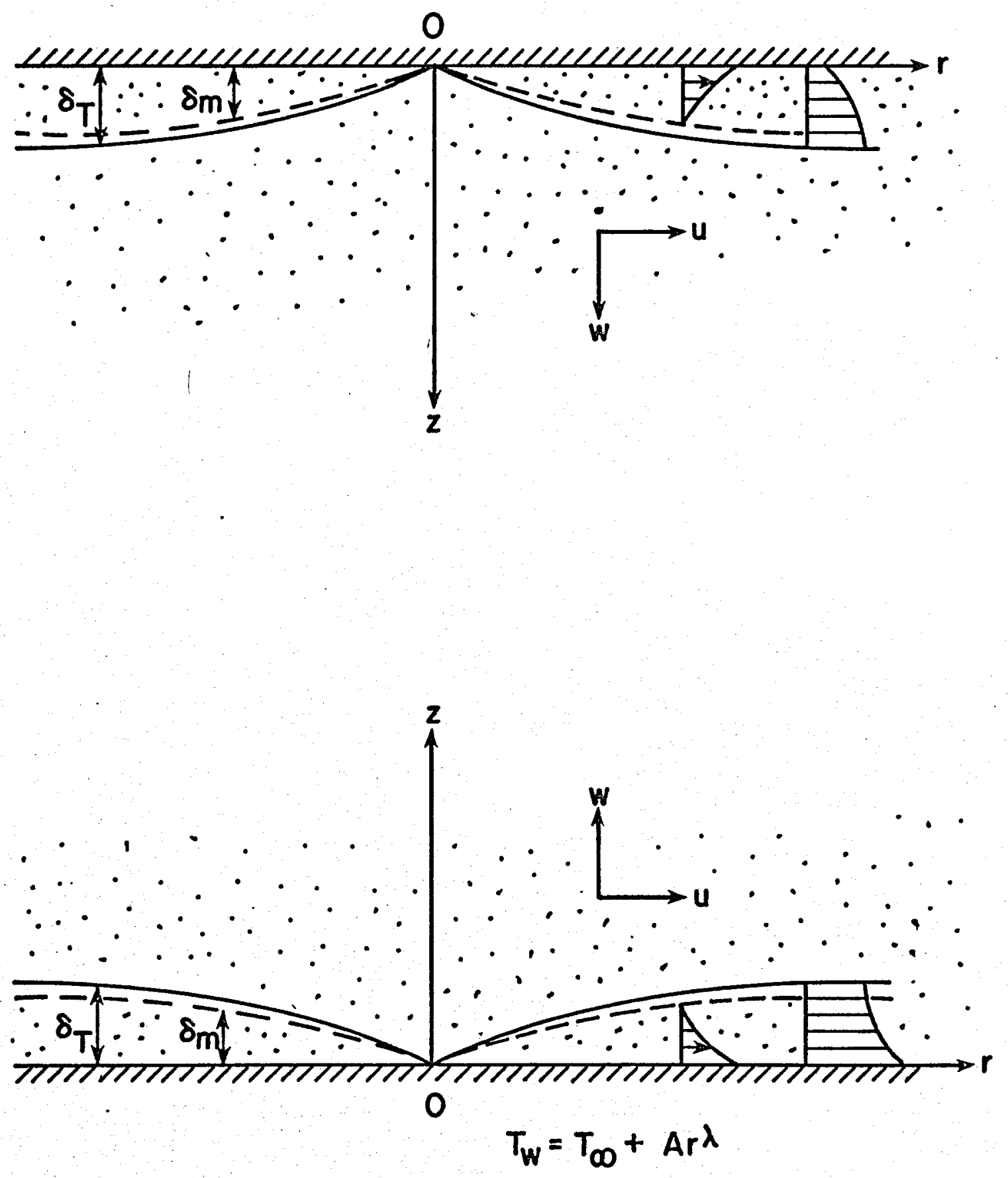

Fig. 1 Coordinate System 


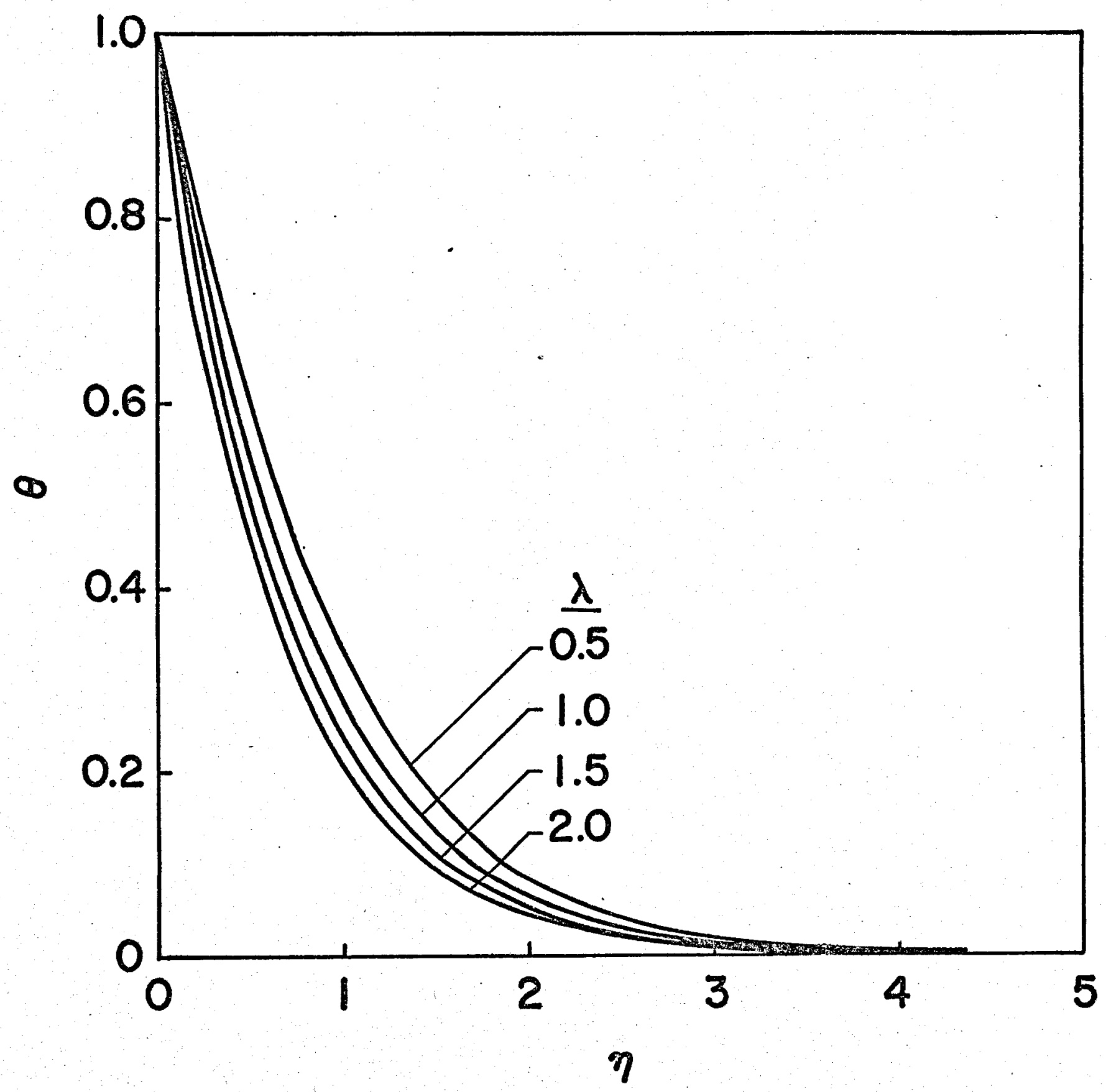

Fig. 2 Values of $\theta$ Versus $n$ for Selected Values of $\lambda$ 


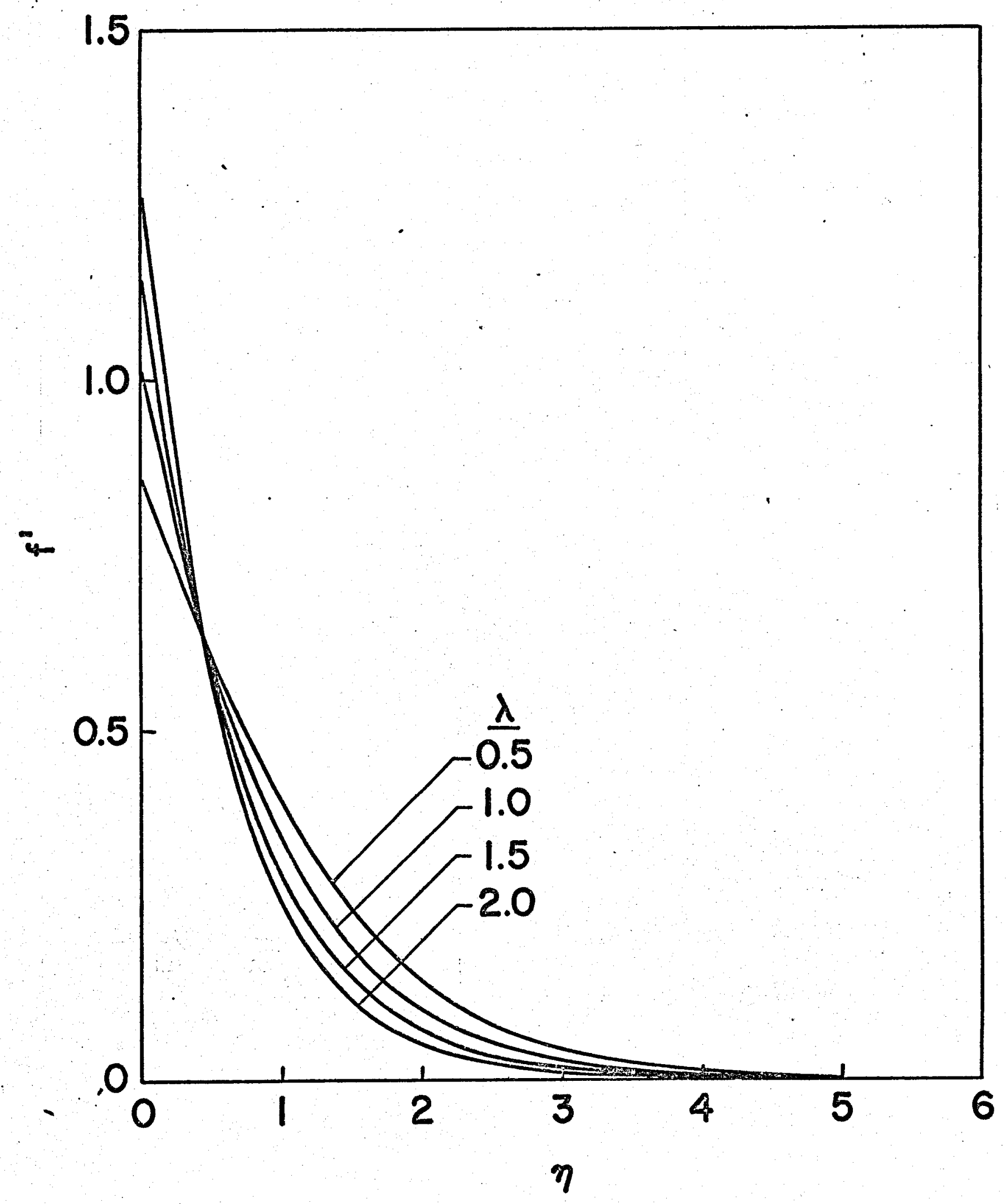

Fig. 3 Dimensionless Velocity Distribution Versus $n$ for Selected Values of $\lambda$ 


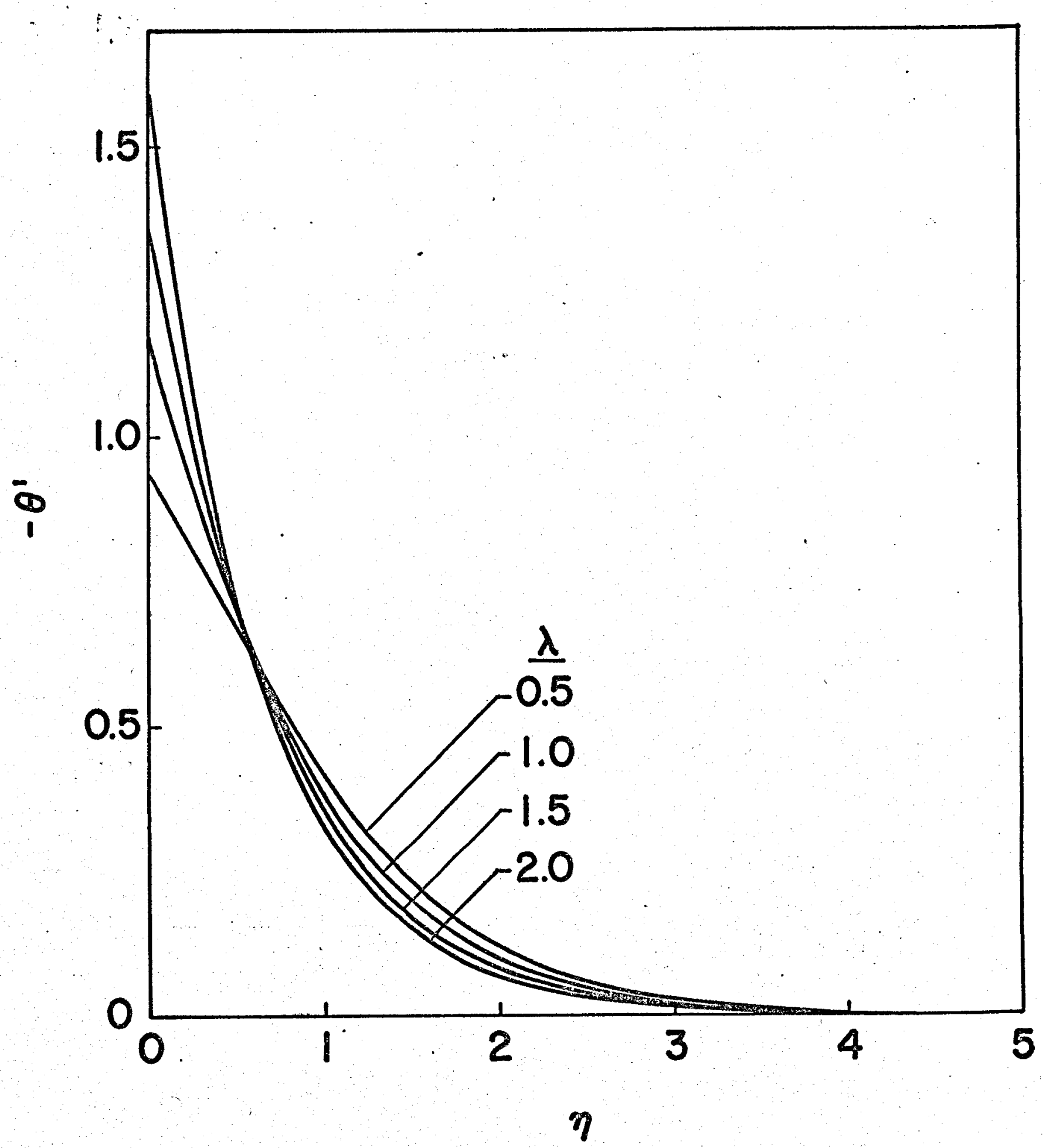

Fig. 4 Values of $-\theta^{\prime}$ Versus $n$ for Selected Values of $\lambda$ 


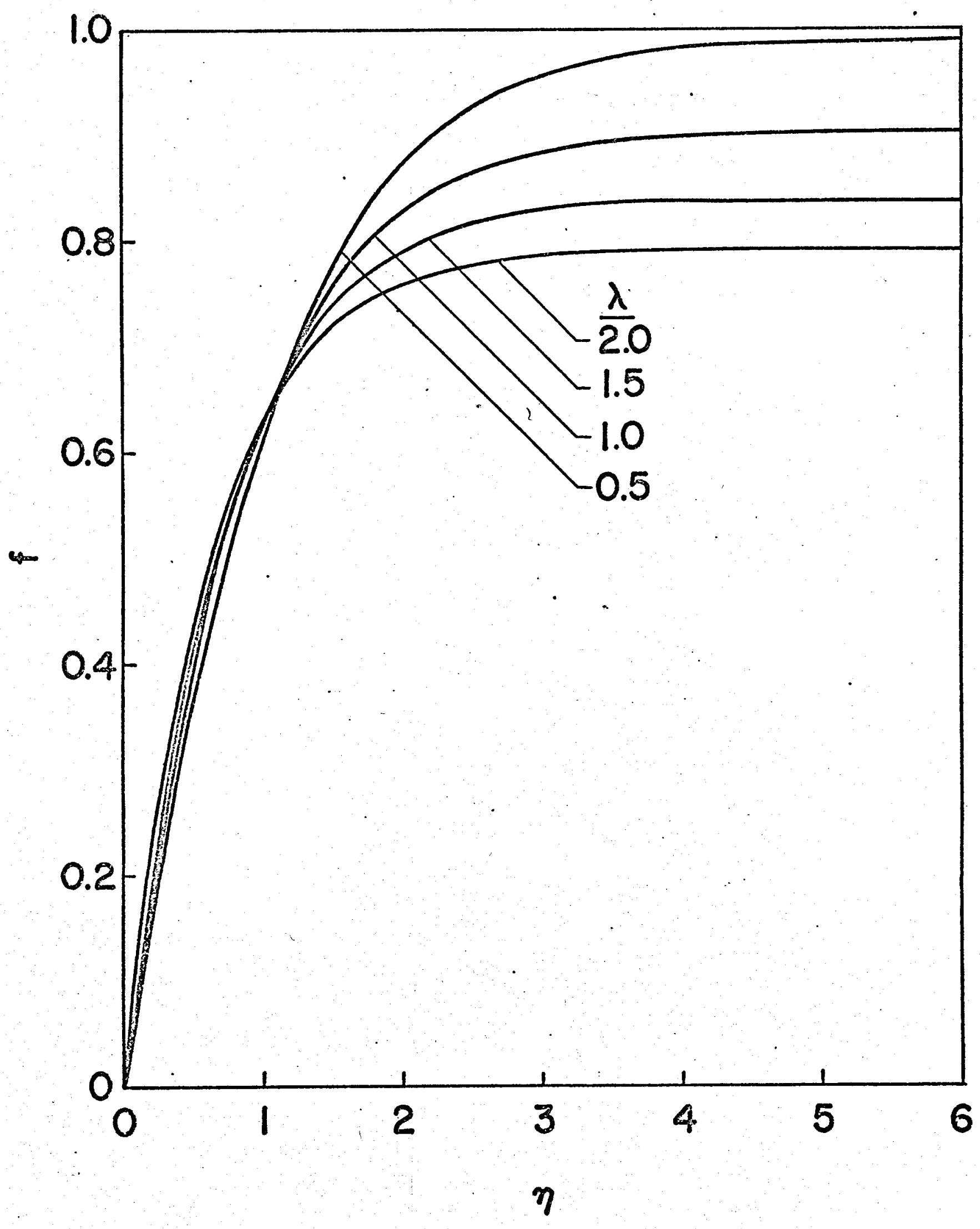

Fig. $5 f$ Versus $\eta$ for Selected Values of $\lambda$ 


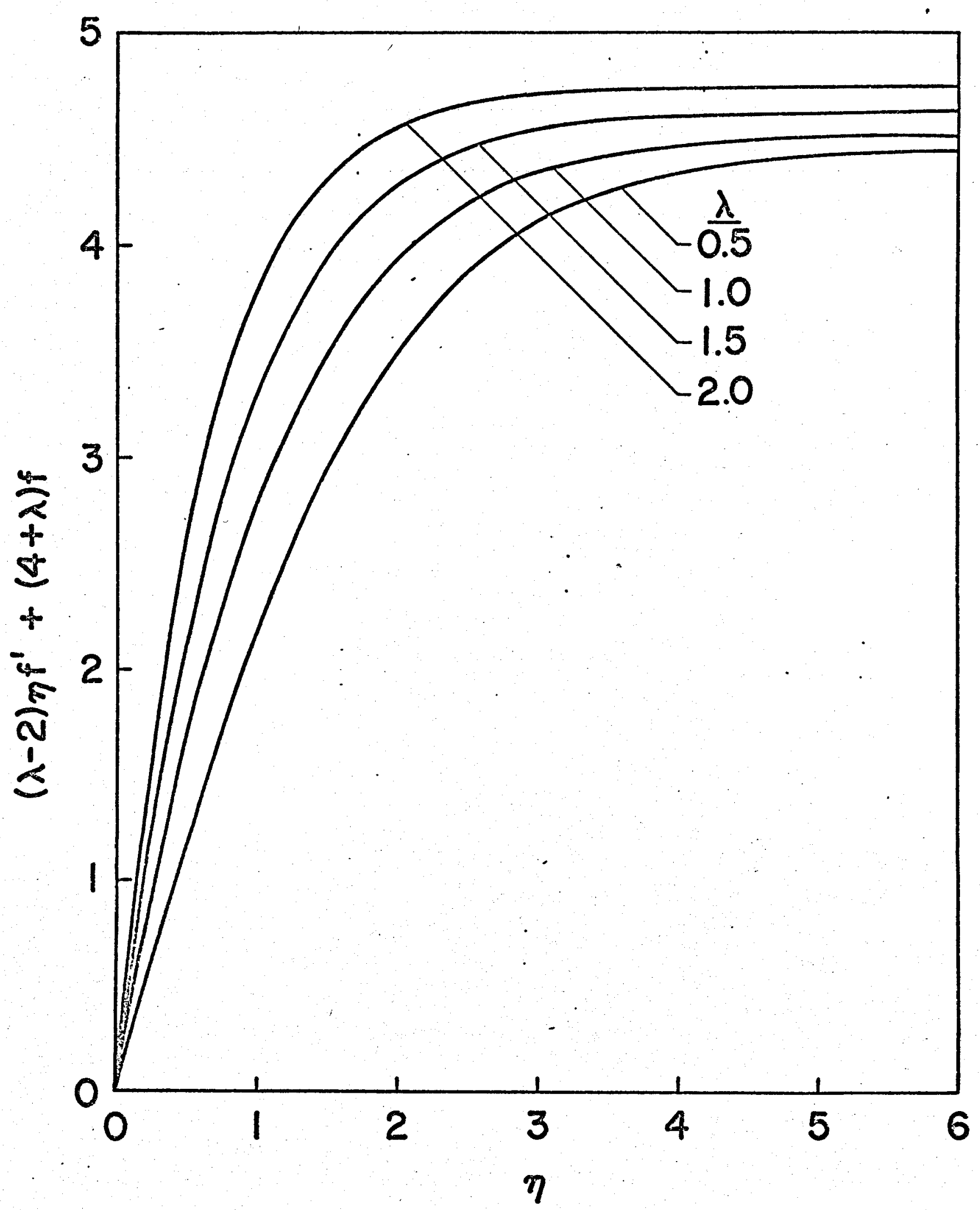

Fig. 6 Dimensionless Vertical Velocity Versus $n$ for Selected Values of $\lambda$. 


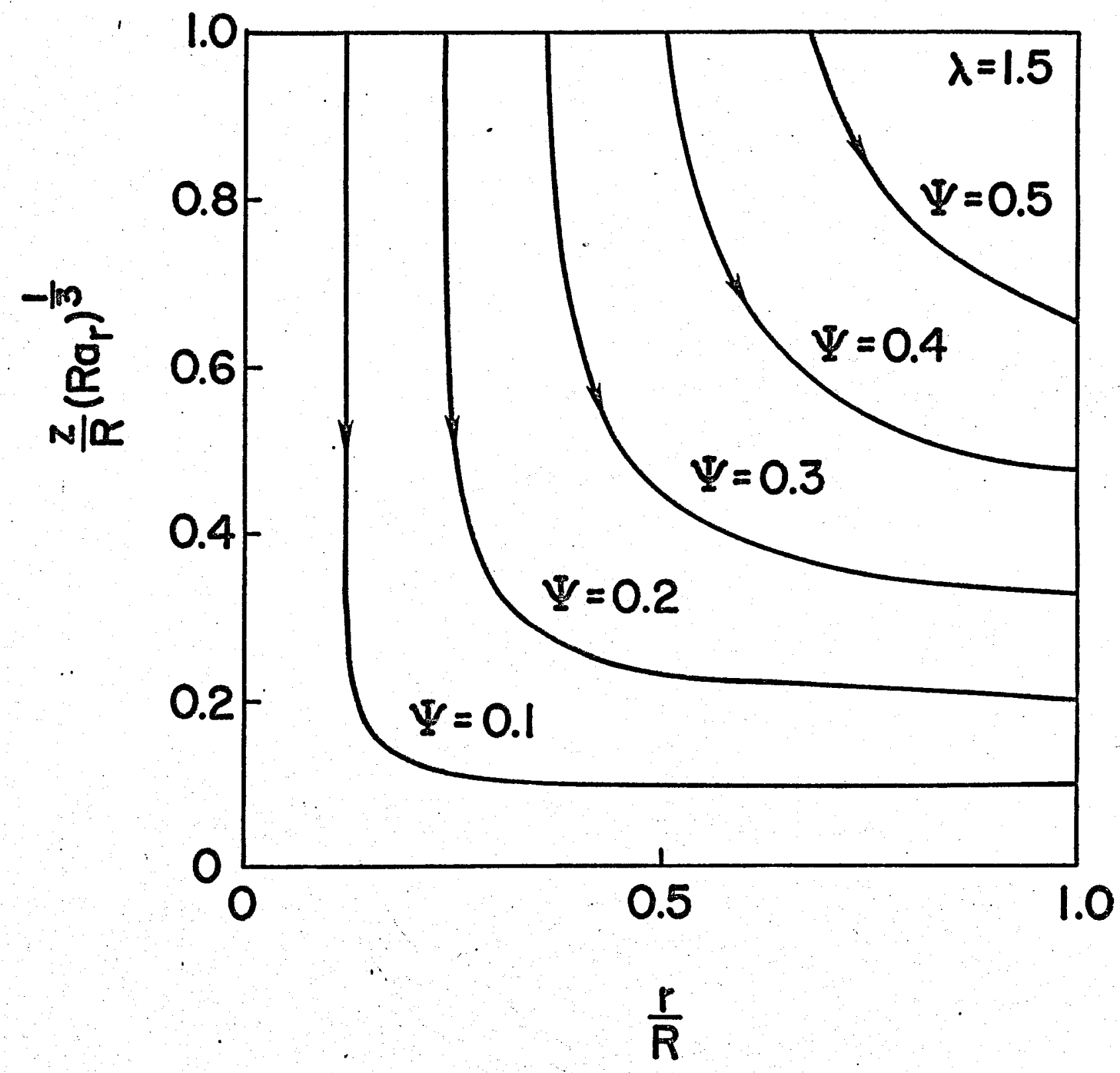

Fig. 7 Streamlines Distribution for a Heated Impermeable Surface Facing Upward 


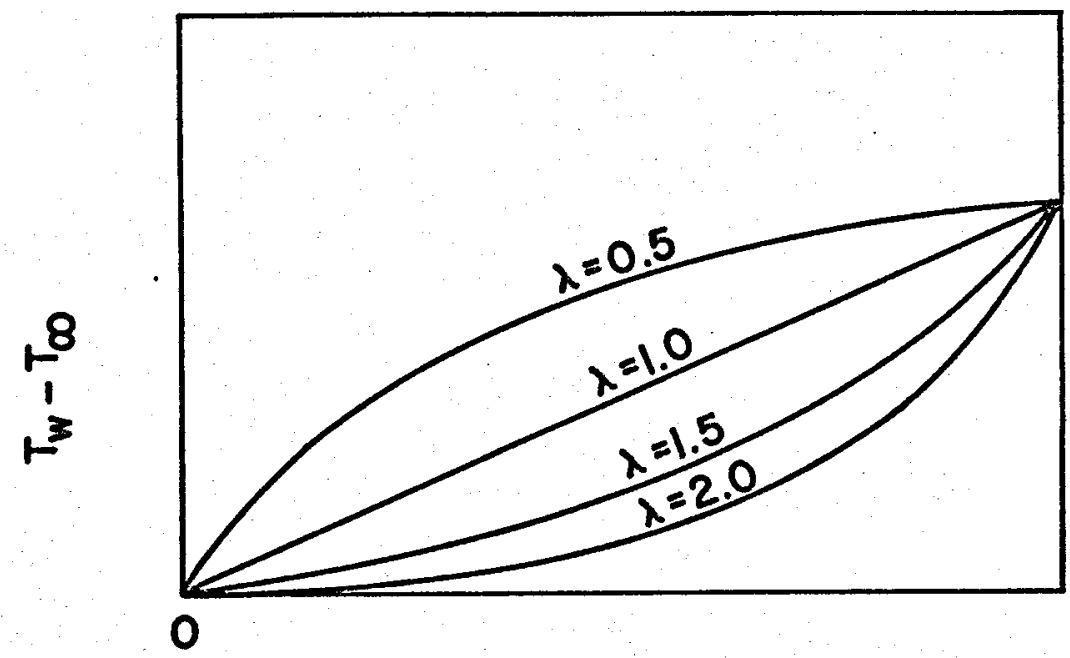

Fig. 8 Sketch of the Wall Temperature Distribution for Selected Values of $\lambda$ 